\title{
Bicuspid aortic valve configuration and aortopathy pattern might represent different pathophysiologic substrates
}

\author{
Fabian A. Kari, MD, ${ }^{\text {a }}$ Shafi S. Fazel, MD, PhD, ${ }^{\mathrm{a}, \mathrm{b}, \dagger} \mathrm{R}$. Scott Mitchell, MD, ${ }^{\mathrm{a}}$ \\ Michael P. Fischbein, MD, PhD, ${ }^{a}$ and D. Craig Miller, MD, ${ }^{a}$ Stanford, Calif, and Toronto, Ontario, Canada
}

The interindividual variability of pathologic bicuspid aortic valve (BAV) configuration is increasingly recognized. The 2007 classification system of Sievers and Schmidtke ${ }^{1}$ describes this diversity of BAV cusp and commissure morphologic characteristics in an unambiguous fashion, thereby facilitating clear communication regarding pathologic BAV cusp configuration. There are numerous associated cardiovascular malformations, but the most common associated problems are ascending aortic and arch dilation.

Four different clusters of BAV-associated aortopathy were identified by cluster analysis of computed tomographic or magnetic resonance thoracic aortic images and introduced by Fazel and associates ${ }^{2}$ in 2008 . The relationships between valvular configuration and pattern of BAV aortopathy, however, were unclear. Valve configuration and aortopathy pattern might be linked to hemodynamic conditions resulting in different distribution and extent of shear and/or stretch along the anterolateral aspect of the ascending aortic wall. Alternatively, there could be phenotypic variability related to specific gene mutations leading to various BAV cusp configuration types being associated with specific aortopathy patterns.

Fazel and associates ${ }^{2}$ did not identify an association between type of valve configuration and aortopathy pattern in 64 patients with a BAV. We updated their assessment using $127 \mathrm{BAV}$ patients according to the Sievers BAV type and Stanford Fazel BAV aortopathy clusters (I-IV). The Sievers BAV type was determined intraoperatively, and aortopathy pattern was based on assessment of gated, thin-slice computed tomographic imaging with 3-dimensional reconstruction of the aortic root, tubular ascending aorta, and aortic arch by 2 independent observers.

Inasmuch as both valve configuration and Fazel cluster data (Figure 1) failed normality tests (Kolmogorov-

\footnotetext{
From the Department of Cardiothoracic Surgery, ${ }^{\mathrm{a}}$ Stanford University Medical School, Stanford, Calif, and Division of Cardiac Surgery, ${ }^{\mathrm{b}}$ University of Toronto, Toronto, Ontario, Canada.

Disclosures: Authors have nothing to disclose with regard to commercial support. ${ }^{\dagger}$ Dr Fazel died in January 2010 after a valiant fight against cancer.

Received for publication Feb 28, 2012; revisions received April 16, 2012; accepted for publication May 15, 2012; available ahead of print June 14, 2012.

Address for reprints D. Craig Miller, MD, Stanford University Medical School, Department of Cardiothoracic Surgery, Falk Cardiovascular Research Center, Stanford, CA 94305-5407 (E-mail: dcm@ stanford.edu).

J Thorac Cardiovasc Surg 2012;144:516-7

$0022-5223 / \$ 36.00$

Copyright (C) 2012 by The American Association for Thoracic Surgery doi: $10.1016 /$ j.jtcvs.2012.05.035
}

Smirnov, Shapiro-Wilk, $P<.05)$, nonparametric statistical measures were used to judge the strength of association between certain BAV types and aortopathy clusters (formal normalization of data was unsuccessful). The null hypothesis of no difference between groups defined by the Sievers valve configuration or between the Fazel BAV aortopathy clusters was also tested. We wanted to clarify this question: Is there any kind of relation between BAV configuration and localization/extent of the aortopathy?

Neither correlation nor analysis of variance results was significant: Spearman correlation was not significant for any pair of Fazel cluster and Sievers cusp configuration types $(P>.05)$. When patients were assigned to different groups according to Sievers valve type, analysis of variance on ranks test showed no significant differences in aortopathy clusters $(P=.543)$. Comparison of groups according to Fazel aortopathy cluster using the same test revealed no significant difference when tested for Sievers cusp type $(P=.111)$.

Subjectively, a distinctive fifth type of aortic morphologic disease not captured completely by our earlier quantitative, objective cluster analysis was noted frequently by both observers. This cluster included enlargement of the aortic root (sinuses of Valsalva) and the tubular ascending aortic segment (combination of Fazel clusters I and II). We describe this finding as cluster "Ia," with preserved sinotubular junction and normal tubular ascending aortic segment (formerly, Fazel cluster I), and cluster "Ib," in which the sinotubular junction is effaced and the tubular ascending aorta is dilated (Figure 1). This impression requires validation in larger series of BAV patients. It should be noted that patients with isolated single-sinus aneurysm with or without ascending aortic aneurysm are currently not captured by any of the Fazel clusters. Future studies should focus on this specific, interesting patient substrate.

Debate continues about the impact of genetically determined intrinsic disease of the aortic wall, as opposed to altered hemodynamics increasing shear and stretch on the aortic wall, leading to progression of aortopathy. Hypothetically, hemodynamic sequelae of different BAV types might lead to different levels of aortic wall strain in different aortic regions. A significant association between Sievers type 1/L- R ("majority type") cusp configuration and more severe degree of wall degeneration in the ascending aorta compared with Sievers 1/R-N ("minority type") and $1 / \mathrm{N}-\mathrm{L}$ valves has been described. ${ }^{3}$ Valve function might be of importance, although not tested in detail in this study. 

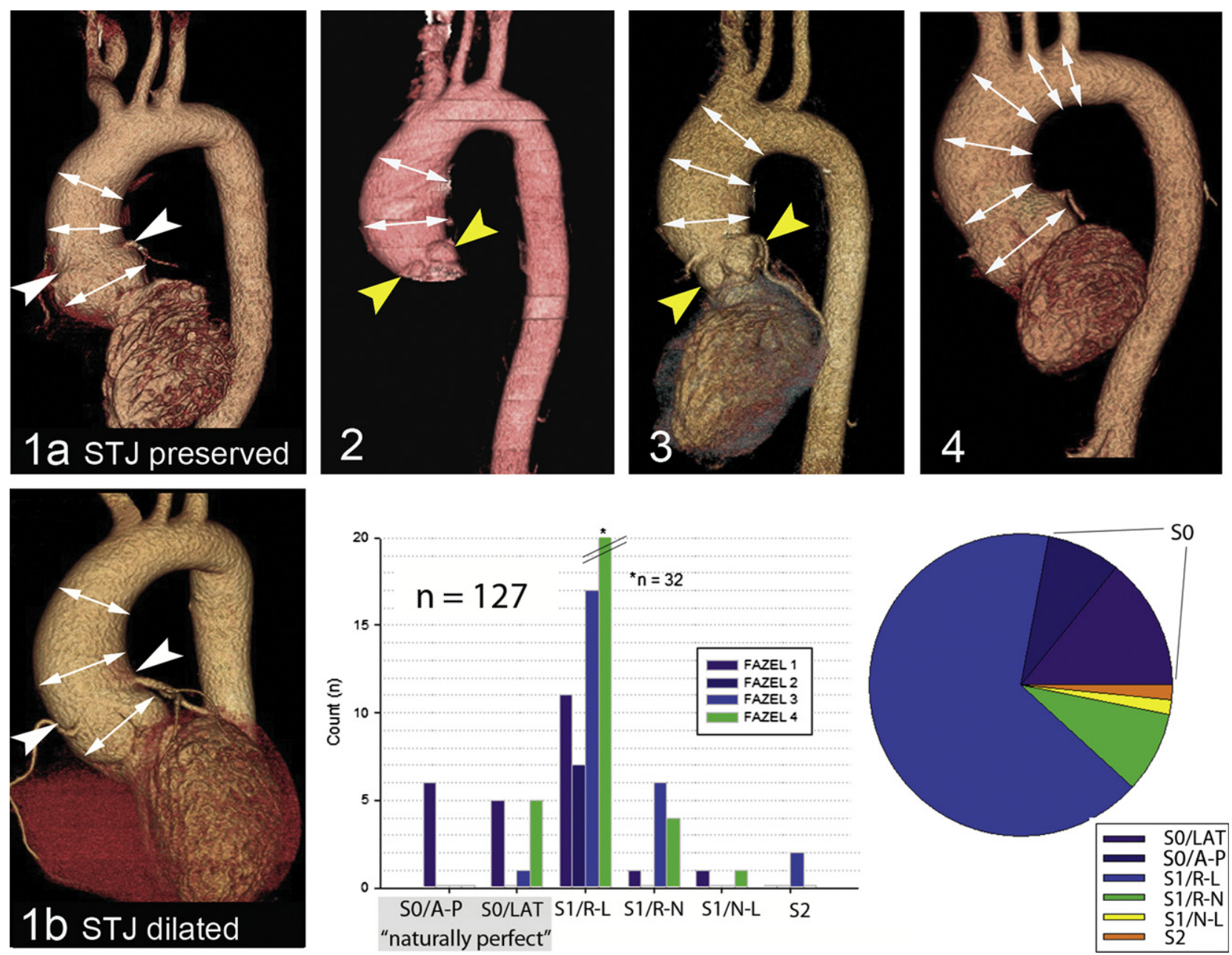

FIGURE 1. Four types of aortopathy (white numbers) originally identified by cluster analysis of 64 patients (2) and modification of the original cluster 1 (now 1a and 1b). White double arrows, dilated aortic segments. White arrowheads, Sinotubular junction (STJ) preserved/dilated in clusters 1a and 1b. Yellow arrowheads, Normal sinuses in clusters 2 and 3. The two inlays give an overview over prevalence of Sievers types and Fazel clusters in this series of patients.

However, valve function has been shown to correlate with qualitative severity of aortopathy. ${ }^{4}$ Acute outflow jet angles from the left ventricular outflow tract correlate with increased circulating levels of matrix metalloproteinase-2 and matrix metalloproteinase- 9 and with more severe aortic dilation ${ }^{5}$; on the other hand, even normal tricuspid aortic valves can be associated with stiffer, less compliant, and enlarged thoracic aortic walls in first-degree relatives of patients with a BAV. Aortic dilation can progress after isolated BAV replacement, which could portend more subsequent aortic complications.

Our data suggest that the location and the extent of aortopathy, described as Fazel-Stanford clusters, are not directly interrelated to BAV configuration. As a consequence, unlike local qualitative medial disease, aortopathy cluster is probably not predetermined by hemodynamics. This implies the possibility of cusp fusion pattern on the one hand and longitudinal extent and arch involvement of the aortopathy on the other hand, representing different pathophysiologic substrates.

\section{References}

1. Sievers HH, Schmidtke C. A classification system for the bicuspid aortic valve from 304 surgical specimens. J Thorac Cardiovasc Surg. 2007;133: 1226-33.

2. Fazel SS, Mallidi HR, Lee RS, Sheehan MP, Liang D, Fleischman D, et al. The aortopathy of bicuspid aortic valve disease has distinctive patterns and usually involves the transverse aortic arch. J Thorac Cardiovasc Surg. 2008; 135:901-7.e1-2.

3. Russo CF, Cannata A, Lanfranconi M, Vitali E, Garatti A, Bonacina E. Is aortic wall degeneration related to bicuspid aortic valve anatomy in patients with valvular disease? J Thorac Cardiovasc Surg. 2008;136:937-42.

4. Roberts WC, Vowels TJ, Ko JM, Filardo G, Hebeler RF Jr, Henry AC, et al. Comparison of the structure of the aortic valve and ascending aorta in adults having aortic valve replacement for aortic stenosis versus for pure aortic regurgitation and resection of the ascending aorta for aneurysm. Circulation. 2011;123: 896-903.

5. den Reijer PM, Sallee D 3rd, van der Velden P, Zaaijer ER, Parks WJ, Ramamurthy $\mathrm{S}$, et al. Hemodynamic predictors of aortic dilatation in bicuspid aortic valve by velocity-encoded cardiovascular magnetic resonance. J Cardiovasc Magn Reson. 2010;12:4. 